\title{
SAR Deception Jamming Target Recognition Based on the Shadow Feature
}

\author{
Xinxin Tang, Xiaoling Zhang, Jun Shi, Shunjun Wei, Lei Yu \\ University of Electronic Science and Technology of China \\ Chengdu, Sichuan, China \\ Email: 358520002@qq.com
}

\begin{abstract}
SAR deception jamming method is one of the most important jamming techniques by overlapping a group of fake targets into the SAR images, which can greatly reduce the accuracy of the SAR image interpretation. On the other hands, as a kind of active remote sensing technique, SAR system has less diffuse scattering, and the shadow characteristic is more significant than the optic system. In this paper, the shadow characteristics of the true and false targets are discussed via the simulation experiment, and the convolutional neural network(CNN) is applied for SAR deception jamming target recognition based on the shadow feature. Numerical experiments have shown that the CNN method can effectively distinguish the true and false targets correctly through the shadow feature.
\end{abstract}

Index Terms-Deception targets, SAR, CNN, Shadow Feature.

\section{INTRODUCTION}

Synthetic aperture radar(SAR) can get high-resolution images under bad weather conditions and complex environments at all time [1]. With its increasing application in military field, some jamming methods are also developed to prevent the targets from being detected,classified,and recognized by the SAR [2]-[4]. The deception jamming method realizes its jamming function by modulating and retransmitting the intercepted SAR signals [5]. So the deception jammer can produce false scenes in the real SAR images by transmitting the coherent jamming signals with comparatively lower power. The false targets fabricated by the deception jammer will seriously affect SAR images' interpretation, and let the SAR system make inaccurate classification decisions. Thus, providing some strategies to recognise these false targets is very necessary.

Even though the false scene produced by a deception jammer is of close resemblance with the real one, an obvious difference exists between them - the shadow feature. Thanks to the active remote sensing technology, SAR system has less diffuse scattering phenomenon than the optical system. So images created by the SAR system have more obvious shadow feature. In fact, shadow feature has already been utilized in SAR domain. The combination of a target's scattering characteristic and its shadow feature can describe the outline of a target more clearly. And the target recognition of joint shadow has become an important method for SAR image interpretation [6], [7]. Theoretically, the deception jamming targets staggered with the real scenes can't have the shadow features. So we can recognize the true targets and the false targets successfully with their shadow feature.

Recently, convolutional neural network $(\mathrm{CNN})$, one of the deep learning architectures, has been successfully used in image classification, object detection, action recognition etc [8], [9]. The CNN's classification performance can be divided into two steps: feature extraction in convolutional layers and object classification in softmax layer. With weights sharing mechanism and convolution operations of CNN, It is widely used in image recognition. One effective result achieved by CNN is in the 2012 ImageNet data set, where they lower the error rate in the test data set from the previous 26.2 percent to the 15.3 percent. This success has fully demonstrated that the CNN has strong capacity in image classification [10].

In this paper, due to a one stage CNN model is unable to achieve an ideal classification result, a two stage CNN model based on the shadow features is proposed to reach a high accuracy for the deception jamming target recognition. The simulation experiments have shown that our method can distinguish the true and false targets correctly.

The organization of this paper is as follows: section II describes the formation mechanism of targets' shadow feature. Section III introduces the CNN's principle and proposes the structure of our CNN. In section IV, some simulation experiments are performed to validate the efficiency of the proposed method. Section V concludes this paper.

\section{Shadow Feature}

The deception jamming method affects SAR images' interpretation through adding the deception targets' information into the SAR echoes. The SAR sensor of vertical side-looking strip-mode is assumed flying along an ideal straight track with a constant velocity $v$, as shown in Fig.1.

The $y$-axis is parallel to the track of the SAR. The $z$-axis is perpendicular to the ground and positive upward. The $x$ axis is determined by the right-hand rule. Point $S$ represents the SAR sensor which is located in $(0,0, H)$ at the zero moment. Point $A_{1}$ is a jammer, and point $A_{2}$ is the location of the deception jamming point target. Their coordinates are denoted by $\left(x_{j}, y_{j}, 0\right)$ and $\left(x_{i}, y_{i}, 0\right)$ respectively. The jammer transmits the modulated signal captured from the SAR to fabricate the false targets into the real SAR scenes, which can hinder SAR images' interpretation. From the above geometric relations, we can know 


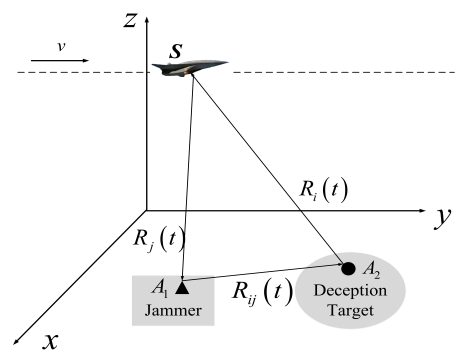

Fig. 1. Model of deception jamming

$$
\begin{aligned}
& R_{j}(t)=\sqrt{H^{2}+x_{j}^{2}+\left(v t-y_{j}\right)^{2}} \\
& R_{i}(t)=\sqrt{H^{2}+x_{i}^{2}+\left(v t-y_{i}\right)^{2}}
\end{aligned}
$$

SAR transmits the linear frequency modulation signal whose mathematical expression is

$$
s(\tau)=g(\tau) \exp \left(j 2 \pi f_{0} \tau\right) \exp \left(j \pi k \tau^{2}\right)
$$

where $g(\tau)$ is rectangular signal, $k$ is the frequency modulation rate and $f_{0}$ is centre frequency. The fourier transform of $s(\tau)$ is denoted as $s(\omega)$. The echo of the deception point target $A_{2}$ can be written as

$$
\begin{aligned}
s_{i}(\tau)= & \delta_{i} s\left(\tau-\frac{2 R_{i}(t)}{c}\right)=\delta_{i} g\left(\tau-\frac{2 R_{i}(t)}{c}\right) \\
& \times \exp \left(j 2 \pi f_{0}\left(\tau-\frac{2 R_{i}(t)}{c}\right)\right) \\
& \times \exp \left(j \pi k\left(\tau-\frac{2 R_{i}(t)}{c}\right)^{2}\right)
\end{aligned}
$$

its corresponding fourier transform is

$$
\begin{gathered}
x_{i}(\omega, t)=s(\omega) \delta_{i} \exp \left(-j 2 \pi f_{0} T_{i}(t)\left(1+\frac{\omega}{\omega_{0}}\right)\right) \\
T_{i}(t)=\frac{2 R_{i}(t)}{c}
\end{gathered}
$$

in the same way, the spectrum of point $A_{1}$ where the jammer is can also be written as

$$
\begin{gathered}
x_{j}(\omega, t)=s(\omega) \delta_{j} \exp \left(-j 2 \pi f_{0} T_{j}(t)\left(1+\frac{\omega}{\omega_{0}}\right)\right) \\
T_{j}(t)=\frac{2 R_{j}(t)}{c}
\end{gathered}
$$

where $T_{i}(t)$ and $T_{j}(t)$ represent the echo delay of point $A_{2}$ and point $A_{1}$ respectively.

In order to produce the echo of a deception jamming point target in a specific location, the jammer's system corresponding function should be

$$
\begin{gathered}
H(\omega, t)=\delta_{i} \exp \left(-j 2 \pi f_{0} \Delta T_{i j}(t)\left(1+\frac{\omega}{\omega_{0}}\right)\right) \\
\Delta T_{i j}=T_{i}(t)-T_{j}(t)
\end{gathered}
$$

In the equation (9), $\delta_{i}$ is the back scattering coefficient of the deception jamming point target and $\Delta T_{i j}$ is the system response time of the jammer. From the equation (10), we can

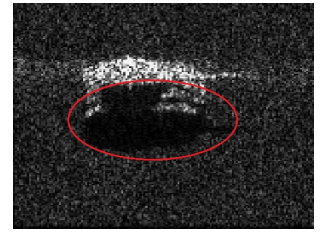

(a)

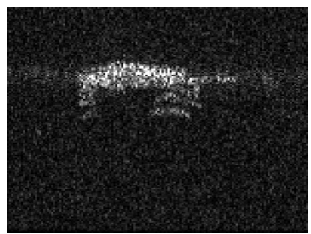

(b)
Fig. 2. Simulation targets.(a)real tank (b)deception tank

know the jammer can make the deception jamming point target in different locations through adjusting $\Delta T_{i j}$. So the equation (5) can be written as

$$
x_{i}(\omega, t)=x_{j}(\omega, t) H(\omega, t)
$$

The echoes received by the SAR are composed of the true target signal and the deception target signal, whose spectrum can be expressed as follows:

$$
z(\omega, t)=x(\omega, t)+x_{j}(\omega, t)
$$

where $x(\omega, t)$ is the spectrum of the true SAR target. From the equation (12), we can know the deception target is overlapped in the real SAR scene. Consequently, neither does it have the geometrical condition to generate the shadow, nor can it cut the signal strength nearby to create the shadow. From Fig.2, we can see the true tank has a more significant shadow feature (the area marked with a red elliptical curve) than the deception tank. Thus, we can distinguish the true and false targets with their shadow feature.

\section{Convolutional Neural Network}

Convolutional neural network(CNN) is the multi-layer neural network including the input layer, the convolutional layer, the subsampling layer and the fully connected classification layer. Each layer is composed of many independent neurons. Multi-channel image data $\boldsymbol{x} \in \boldsymbol{R}^{h \times w \times c}$ as the input of the convolutional neural networks will be transformed to output map $\boldsymbol{y} \in \boldsymbol{R}^{h^{\prime} \times w^{\prime} \times c^{\prime}}$ after the convolution operation in the convolution layer, where $h, w, c$ denote the height, width, and dimension of the image data.

$y_{i^{\prime} j^{\prime} k^{\prime}}=f\left(\sum_{i=1}^{h^{\prime}} \sum_{j=1}^{w^{\prime}} \sum_{m=1}^{c} K_{i j m k^{\prime}} x_{i^{\prime}+i, j^{\prime}+j, m}+b_{k^{\prime}}\right)$

In equation (13), $K \in \boldsymbol{R}^{h^{\prime \prime} \times w^{\prime \prime} \times c^{\prime \prime} \times c^{\prime}}$ represents convolution kernel, $b_{k}^{\prime}$ is a bias after a convolution operation, and $f(\cdot)$ denotes a nonlinear activation function called the rectified linear unit (ReLU) [11] which is shown as follows

$$
f(x)=\max (0, x)
$$

After the convolution operation, we use the dropout method to prevent neural networks from overfitting [12]. Then, we subsample the feature maps in the subsampling layer with the max-pooling operation whose expression is

$$
y_{i^{\prime} j^{\prime} k^{\prime}}=\max _{1<i<h^{\prime \prime \prime}, 1<j<w^{\prime \prime \prime}} x_{i^{\prime}+i, j^{\prime}+j, k}
$$




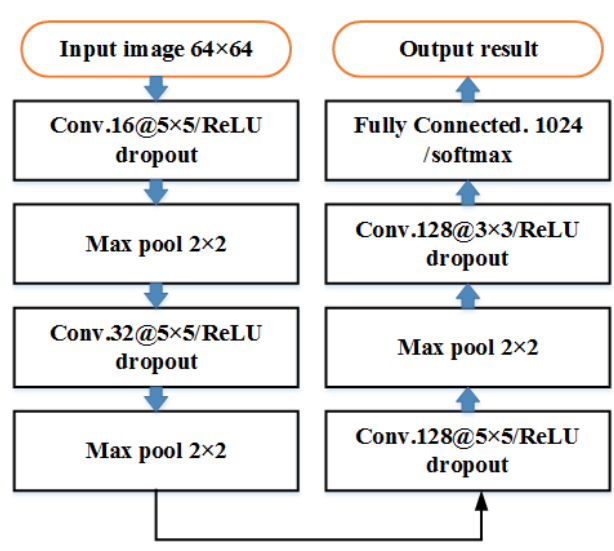

Fig. 3. The structure of our CNN. Conv. represents a convolution layer.

Finally,we apply the softmax classifier in the fully connected layer to finish the classification task. The expression is denoted as

$$
p_{i}=\frac{\exp \left(x_{i j k}\right)}{\sum_{m=1}^{c} x_{i j m}}
$$

The architecture of our first CNN stage can be seen in Fig. 3 where four convolution layers are utilized as the feature extractors, and a softmax classifier is utilized as the multiclass classifier. Each convolution layer is followed by a maxpooling layer. The size of first three convolution filters is $5 \times 5$ (pixel), the last convolution filter is $3 \times 3$ (pixel), and the number of units in the fully connected layer is 1024 . Our second stage of CNN simpler than the former only have the first two convolution layers with one max-pooling layer between them and one fully connected layer with 512 units. We train the parameters of our $\mathrm{CNN}$ with stochastic gradient descent algorithm and back-propagation algorithm.

\section{EXPERIMENT ON SIMULATION DATA SET}

The experiment data used in this paper are collected on the simulation platform. The simulation data set includes four kinds of models: tank1, tank2, rocket artillery and truck which are shown in Fig.4-top. Their SAR imaging results are shown in Fig.4-middle and their corresponding deception targets' imaging results(without the shadow feature) are shown in Fig.4-bottom. These targets are simulated using a Ka-band SAR sensor, in a $0.15 \mathrm{~m}$ resolution strip mode, full aspect coverage (in the range of $1^{\circ}$ to $360^{\circ}$, with the interval of $1^{\circ}$ ). And their backscattering coefficients are simulated with FEKO [13]. Training images are captured at $47^{\circ}$ depression angle, and testing images are acquired at $45^{\circ}$ depression angle. The number of images each class is 360 , and the size of each image is $64 \times 64$ (pixel).

\section{A. Data Preprocessing}

At the beginning, the true targets' training samples and their relevant false targets' training samples are considered as two different classes. That is to say, the true SAR targets and their corresponding deception jamming targets are considered as two different classes. So eight kinds of training samples are used to train the first stage of CNN. The classification result

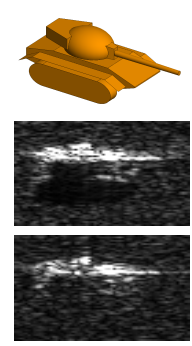

(a)

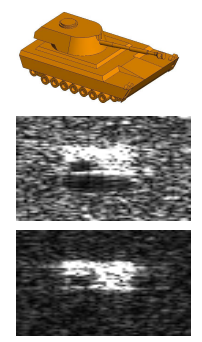

(b)

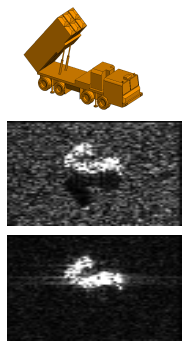

(c)

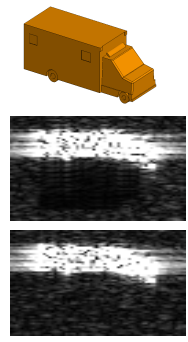

(d)
Fig. 4. Types of simulation targets. (a)tank1 (b)tank2 (c)rocket artillery (d)truck

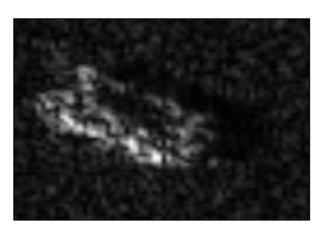

(a)

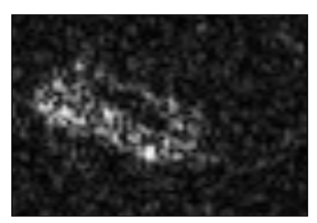

(c)
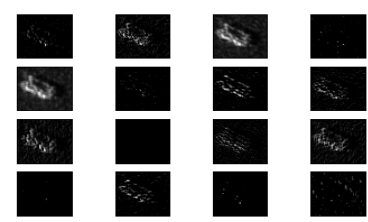

(b)
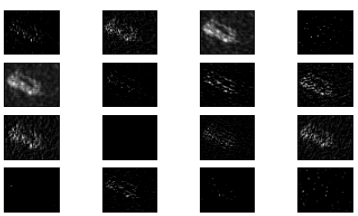

(d)
Fig. 5. Tank1 and its output feature maps in the first convolution layer.(a)true tank1 (b)true tank1's output feature maps(c)false tank1 (d)false tank1's output feature maps

is shown in TABLEI, the accuracies of the true tank 1 and the true truck are only $64.17 \%$ and $51.11 \%$. Many of them are incorrectly classified to their corresponding false targets, which decreases the accuracies.

By comparing the output features of tank1 in Fig.5, there is no obvious difference in the output feature maps between the true tank 1 and the false tank1. This is the reason why the true targets and the false targets can not be distinguished with a one stage CNN. To address this problem, a two stages of CNN is proposed to improve the accuracy of the true and false targets. Inspired by CNN's high accuracy in handwriting recognition where training and testing images can be thought as binary images, SAR images input to the second CNN stage are transformed to the multi-value images. The otsu algorithm [14] is adopted to select suitable threshold value, and the median filtering method together with morphological processing is adopted to segment the SAR images.

For example, the segment results of true tank1 and false tank1 are shown in Fig.6(a) and Fig.7(a), where white represents target, gray represents background clutter and black represents shadow. Their corresponding output feature maps are shown in Fig.6(b) and Fig.7(b) respectively. From these, we can see the true and false tanks' features obtained in the second stage of CNN are of great difference. So, the second CNN stage can learn the shadow feature correctly. 
TABLE I

CONFUSION MATRIX For First Stage CNNs

\begin{tabular}{|c|c|c|c|c|c|c|c|c|c|}
\hline Class & $\begin{array}{c}\text { True } \\
\text { tank1 }\end{array}$ & $\begin{array}{c}\text { False } \\
\text { tank1 }\end{array}$ & $\begin{array}{c}\text { True } \\
\text { tank2 }\end{array}$ & $\begin{array}{c}\text { False } \\
\text { tank2 }\end{array}$ & $\begin{array}{c}\text { True } \\
\text { rocket } \\
\text { artillery }\end{array}$ & $\begin{array}{c}\text { False } \\
\text { rocket } \\
\text { artillery }\end{array}$ & $\begin{array}{c}\text { True } \\
\text { truck }\end{array}$ & $\begin{array}{c}\text { False } \\
\text { truck }\end{array}$ & Accuracy(\%) \\
\hline True tank1 & 231 & 129 & 0 & 0 & 0 & 0 & 0 & 0 & 64.17 \\
\hline False tank1 & 0 & 360 & 0 & 0 & 0 & 0 & 0 & 0 & 100 \\
\hline True tank2 & 0 & 1 & 359 & 0 & 0 & 0 & 0 & 0 & 99.72 \\
\hline False tank2 & 0 & 22 & 0 & 338 & 0 & 0 & 0 & 0 & 93.89 \\
\hline True rocket artillery & 0 & 0 & 0 & 0 & 360 & 0 & 0 & 0 & 100 \\
\hline False rocket artillery & 0 & 0 & 0 & 0 & 6 & 354 & 0 & 0 & 98.33 \\
\hline True truck & 0 & 3 & 0 & 0 & 0 & 0 & 184 & 173 & 51.11 \\
\hline False truck & 0 & 8 & 0 & 0 & 0 & 0 & 0 & 352 & 97.78 \\
\hline Total & & & & & & & 88.13 \\
\hline
\end{tabular}

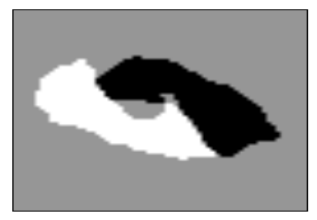

(a)

IIn

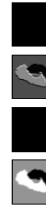

(b)
Fig. 6. Output feature maps of multiple-value real image.(a)multiple-value real image (b)output feature maps

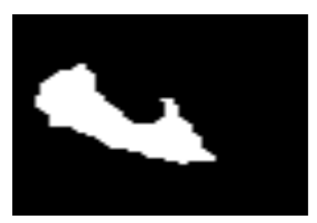

(a)
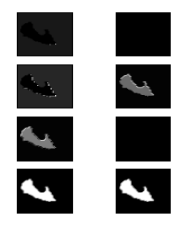

(b)
Fig. 7. Output feature maps of multiple-value fake image.(a)multiple-value fake image (b)output feature maps

\section{B. True and False Targets Classification}

In this paper, a two-stages $\mathrm{CNN}$ is applied to accomplish the classification task of the true targets and the deception jamming targets. First stage is performed as the target recognition classifier that classify four kinds of the targets, where the true targets and their corresponding false targets are regarded as the same class. And second stage is performed as the binary classifier used to classify the true targets and the false targets. To implement the classification task, one four-class classifier and four binary classifiers have been trained. Flow chart of deception jamming target recognition based on shadow

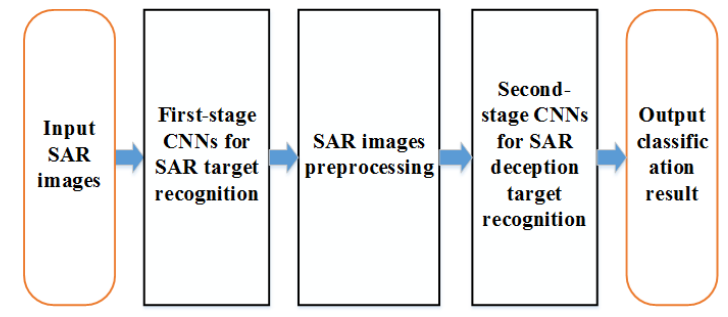

Fig. 8. SAR deception target recognition flow feature is shown in Fig.8. First, the raw SAR images are input to the first CNN stage which have already been trained for SAR target recognition. Next, the image preprocessing method for highlighting the shadow feature is applied to the targets classified in the first step. Finally, these preprocessed images are respectively sent to the second CNN stage for SAR deception target recognition. The confusion matrix of the twostage CNN is shown in TABLEII. The accuracies of true tank1 and true truck have increased to $96.39 \%, 100 \%$, and the overall accuracy has also increased to $98.89 \%$.

\section{CONCLUSION}

Using a one-stage CNN to classify the true targets and the deception jamming targets can not acquire a satisfying result. So, a two-stage CNN for promoting the recognition accuracy is demonstrated in this paper. First CNN stage is used for the target recognition, while the second stage of $\mathrm{CNN}$ is used for the true and false targets classification. The simulation results have shown that our approach can reach a high accuracy in the SAR deception jamming target recognition.

\section{ACKNOWLEDGMENT}

This work was supported by the National Natural Science Foundation of China under Grant 61501098,the China Postdoctoral Science Foundation Funded Project under Grant 2015M570778 and the Fundamental Research Funds for the Central Universities under Grant ZYGX2016KYQD107.

\section{REFERENCES}

[1] I. G. Cumming and F. H. Wong, Digital processing of synthetic aperture radar data: algorithms and implementation. Artech house, 2005.

[2] W. Xiong, J. Zhong, and L. Cao, "An approach of bridge detection over water in high resolution sar image," in Radar Conference 2013, IET International, pp. 1-4, IET, 2013.

[3] F. Zhang, Y. Shao, Z. Wan, and X. Zhang, "Interpretation of buildings in high resolution sar images based on electromagnetic method," in Geoscience and Remote Sensing Symposium (IGARSS), 2010 IEEE International, pp. 2727-2730, Ieee, 2010.

[4] R. Guida, A. Iodice, and D. Riccio, "Height retrieval of isolated buildings from single high-resolution sar images," IEEE Transactions on Geoscience and Remote Sensing, vol. 48, no. 7, pp. 2967-2979, 2010.

[5] Y. Lee, J. Park, W. Shin, and K. Lee, "A study on jamming performance evaluation of noise and deception jammer against sar satellite," in Synthetic Aperture Radar (APSAR), 2011 3rd International Asia-Pacific Conference on, pp. 1-3, IEEE, 2011. 
TABLE II

Confusion Matrix For The Second Stage Of CNN

\begin{tabular}{|c|c|c|c|c|c|c|c|c|c|}
\hline Class & $\begin{array}{c}\text { True } \\
\text { tank1 }\end{array}$ & $\begin{array}{c}\text { False } \\
\text { tank1 }\end{array}$ & $\begin{array}{c}\text { True } \\
\text { tank2 }\end{array}$ & $\begin{array}{c}\text { False } \\
\text { tank2 }\end{array}$ & $\begin{array}{c}\text { True } \\
\text { rocket } \\
\text { artillery }\end{array}$ & $\begin{array}{c}\text { False } \\
\text { rocket } \\
\text { artillery }\end{array}$ & $\begin{array}{c}\text { True } \\
\text { truck }\end{array}$ & $\begin{array}{c}\text { False } \\
\text { truck }\end{array}$ & Accuracy(\%) \\
\hline True tank1 & 347 & 0 & 10 & 0 & 1 & 0 & 2 & 0 & 96.39 \\
\hline False tank1 & 0 & 360 & 0 & 0 & 0 & 0 & 0 & 0 & 100 \\
\hline True tank2 & 0 & 0 & 358 & 0 & 2 & 0 & 0 & 0 & 99.44 \\
\hline False tank2 & 0 & 0 & 0 & 360 & 0 & 0 & 0 & 0 & 100 \\
\hline True rocket artillery & 0 & 0 & 0 & 0 & 360 & 0 & 0 & 0 & 100 \\
\hline False rocket artillery & 0 & 0 & 0 & 0 & 0 & 360 & 0 & 0 & 100 \\
\hline True truck & 0 & 0 & 0 & 0 & 0 & 0 & 360 & 0 & 100 \\
\hline False truck & 0 & 0 & 0 & 0 & 0 & 0 & 1 & 359 & 99.72 \\
\hline Total & & & & & & & 98.89 \\
\hline
\end{tabular}

[6] S. Papson and R. M. Narayanan, "Classification via the shadow region in sar imagery," IEEE transactions on aerospace and electronic systems, vol. 48, no. 2, pp. 969-980, 2012.

[7] J. Cui, J. Gudnason, and M. Brookes, "Radar shadow and superresolution features for automatic recognition of mstar targets," in IEEE International Radar Conference, 2005., pp. 534-539, IEEE, 2005.

[8] K. Simonyan and A. Zisserman, "Very deep convolutional networks for large-scale image recognition," arXiv preprint arXiv:1409.1556, 2014.

[9] C. Szegedy, W. Liu, Y. Jia, P. Sermanet, S. Reed, D. Anguelov, D. Erhan, V. Vanhoucke, and A. Rabinovich, "Going deeper with convolutions," in Proceedings of the IEEE Conference on Computer Vision and Pattern Recognition, pp. 1-9, 2015.

[10] A. Krizhevsky, I. Sutskever, and G. E. Hinton, "Imagenet classification with deep convolutional neural networks," in Advances in neural information processing systems, pp. 1097-1105, 2012.

[11] H. B. Sailor and H. A. Patil, "Filterbank learning using convolutional restricted boltzmann machine for speech recognition," in Proc. Speech and Signal Processing (ICASSP) 2016 IEEE Int. Conf. Acoustics, pp. 5895-5899, Mar. 2016.

[12] N. Srivastava, G. E. Hinton, A. Krizhevsky, I. Sutskever, and R. Salakhutdinov, "Dropout: a simple way to prevent neural networks from overfitting.," Journal of Machine Learning Research, vol. 15, no. 1, pp. 1929-1958, 2014.

[13] X. Wang, C. Wang, and Y. Liu, "RCS computation and analysis of target using feko," in Proc. 3rd Asia-Pacific Conf. Antennas and Propagation, pp. 822-825, July 2014.

[14] N. Otsu, "A threshold selection method from gray-level histograms," and Cybernetics IEEE Transactions on Systems, Man, vol. 9, pp. 62-66, Jan. 1979. 